\title{
Educando a los padres argentinos: un análisis a través de los manuales de puericultura de Aráoz Alfaro y Garrahan
}

\section{Educating argentinian parents: an analysis through childcare handbooks written by Aráoz Alfaro and Garrahan}

\author{
Ana Briolotti \\ Consejo Nacional de Investigaciones Científicas y Técnicas \\ Instituto interdisciplinario de estudios de género \\ Universidad Nacional de La Plata \\ (Argentina) \\ anabriolotti@gmail.com
}

\section{Resumen}

El artículo indaga las relaciones establecidas entre la medicina infantil y los saberes psicológicos en la Argentina, focalizándose en la divulgación de conocimientos sobre el desarrollo del niño de primera infancia y su crianza. La puericultura se nutrió de la psicología del desarrollo para orientar a los padres acerca de la educación moral y espiritual de sus hijos, al tiempo que, por medio de tales consejos, intervino en la producción de un cierto tipo de subjetividad considerada normal y saludable. Se analizan dos manuales publicados durante la primera mitad del siglo XX, mostrando similitudes y diferencias en la forma como el discurso médico concibió el desarrollo del niño y procuró intervenir en su curso. En el primer momento analizado, el cuidado del niño debía asegurar su supervivencia y salud física, mientras que en la segunda etapa el objetivo de los padres debía ser garantizar la felicidad del niño y el pleno desenvolvimiento de su personalidad. Estas diferencias están ligadas a ciertos cambios sociopolíticos que tuvieron lugar en la primera mitad del siglo XX e impactaron en la concepción de la propia puericultura, que se reconfiguró promoviendo la adaptación de las pautas de crianza a los rasgos emocionales y psico-sociales del niño.

Palabras Clave: Historia - Medicina - Psicología - Divulgación 


\begin{abstract}
This article discusses the relationship between child medicine and psychological knowledge in Argentina, focusing on the dissemination of scientific knowledge about child's development and upbringing. Childcare drew on the psychology of development to guide parents about moral and spiritual education and through such councils, took part in the production of a certain type of subjectivity considered normal and healthy. Two manuals published during the first half of the 20th century are discussed. They show similarities and differences in the way in which medical discourse conceived child's development and sought to intervene in its course. In the first moment, children care should ensure both survival and physical health, while in the second moment parents were supposed to ensure child's happiness and full development of their personality. These differences are linked to socio-political changes that took place in the first half of the 20th century and had a strong impact on the childcare itself, which was reconfigured to promote guidelines adapted to the psycho-social and emotional traits of the child.
\end{abstract}

Key Words: History - Medicine - Psychology - Dissemination

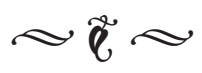

\section{Introducción}

Este artículo indaga las relaciones establecidas entre la puericultura -rama preventiva de la medicina infantil- y los saberes psicológicos en la Argentina, focalizándose en la divulgación de conocimientos sobre el desarrollo del niño de primera infancia y su crianza. Si bien no se trata de una disciplina estrictamente psicológica, el estudio de la puericultura interesa por dos razones: por un lado, porque se nutrió de conocimientos provenientes de la psicología del desarrollo para orientar a los padres en la educación moral y espiritual de sus hijos. Por otro lado, a través de consejos prácticos, intervino en la producción de un cierto tipo de subjetividad considerada normal y saludable. ${ }^{1}$

1. DOS SANTOS LAMPRECHT, Claudia, "Conselhos às mães: manuais de puericultura como estratégia biopolítica na constituição de infâncias saudáveis e normais”,
La puericultura surgió en Europa hacia fines del siglo XIX en estrecha relación con la prevención de enfermedades y la promoción de la salud del niño y de su madre. Una de sus principales líneas de acción fue la divulgación de reglas y preceptos para una crianza considerada "científica", es decir, alejada de las costumbres y saberes populares. Este proyecto educativo puede ser situado en el marco de un proceso más amplio que, de acuerdo con Michel Foucault, se consolidó en el siglo XIX y consistió en la investidura del espacio familiar

en Textura-ulbra, Canoas, 2014, V.XVI, № 32, pp. 210-225. Disponible en: <http://www.periodicos.ulbra. br/index.php/txra/article/view/1252/942> LOREDO NARCIANDI, José Carlos, "La construcción de niños y niñas en España durante el primer tercio del siglo XX: una primera aproximación genealógica”, en CERRI, Chiara \& SÁNCHEZ CRIADO, Tomás (coordinadores) Edades, transiciones e instituciones, Traficantes de sueños, 2014. Disponible en: <http://e-spacio.uned.es/fez/ eserv/bibliuned:500383-IIICongresoEtnografia-1010/ Documento.pdf> 
por parte del saber médico, fundamentalmente a raíz de los peligros atribuidos al autoerotismo infantil. La preocupación por estos riesgos, que remitía en última instancia al interés político y económico por preservar la salud de los futuros ciudadanos, demandó a los padres la vigilancia minuciosa del cuerpo del niño en base a un saber médico-pedagógico. ${ }^{2}$ Este proceso de aculturación médica de los padres, que transformó a la familia en una instancia de normalización del desarrollo infantil ${ }^{3}$, se inscribe en el movimiento de reforma constitutivo de la familia moderna, que consistió en el pasaje del gobierno de las familias al gobierno a través de la familia, entendida como medio (más o menos favorable) para el desarrollo de cada uno de sus miembros. ${ }^{4}$

Es oportuno recordar que si bien los contenidos publicados en los manuales de puericultura tienen un propósito eminentemente informativo, ello no impide analizar su dimensión formativa. En efecto, la difusión del discurso de la puericultura no fue producto del azar, sino que, en paralelo con el proyecto de la institución escolar y médica, se propuso regular todos los actos de la vida, especialmente la de las clases bajas. ${ }^{5} \mathrm{De}$ esta manera, los discursos vinculados con la educación para la salud buscaron difundir un modelo de infancia, de familia y de sociedad

2. FOUCAULT, Michel, El Poder Psiquiátrico, Fondo de Cultura Económica, Buenos Aires, 2005.

3. FOUCAULT, Michel, Los Anormales, Fondo de Cultura Económica, Buenos Aires, 2001.

4. DONZELOT, Jacques, La policía de las familias. Familia, sociedad y poder, Ediciones Nueva Visión, Buenos Aires, 2008.

5. BOLTANSKI, Luc, Puericultura y moral de clase, Editorial Laia, Barcelona, 1974. y pautar ciertas prácticas, actitudes y valores considerados deseables para el progreso de la nación. ${ }^{6}$ Esto permite poner en relación el discurso educativo de la puericultura con los desarrollos de Foucault sobre la biopolítica y su relación con la gubernamentalidad, vale decir, las formas de poder que tomaron como objeto a la población actuando de manera calculada sobre las fuerzas, las acciones y las relaciones de los individuos. ${ }^{7}$ En este marco la puericultura en tanto discurso de subjetivación intervino en el proceso de descentralización del poder por el cual los estados liberales limitaron la coacción directa sobre los individuos y la sustituyeron por la regulación indirecta de sus conductas, deseos y aspiraciones, con el propósito de alcanzar fines socialmente valorados. En este contexto, la autodisciplina y el autogobierno se transformaron en rasgos deseables y la puericultura intervino activamente en la producción de esa subjetividad "normal" por medio de consejos sobre tópicos diversos: cómo alimentar al niño, cómo bañarlo y vestirlo, qué actividades proponerle, cómo y para qué educarlo, entre otros. Dicho de otra manera, cuando el discurso de la puericultura describía lo que el niño era o lo que debía llegar a ser, en rigor de verdad, contribuía a producir un tipo de subjetividad deseable: aquella adaptada a la lógica de las democracias liberales. Si bien no se trata de un rasgo privativo de la puericultura, su caso es especialmente ilustrativo, no sólo por

6. JIMÉNEZ LUCENA, Isabel, RUIZ SOMAVI-
LLA, María José y CASTELLANOS GUERRERO,
Jesús, “Un discurso sanitario para un proyecto político.
La educación sanitaria en los medios de comunicación
de masas durante el primer franquismo", en Asclepio,
Madrid, 2002; V. LIV, No 1, pp. 201-218. Disponible
en: <http://asclepio.revistas.csic.es/index.php/asclepio/
article/view/126/125>

7. FOUCAULT, Michel, Seguridad, territorio, población, Fondo de Cultura Económica, Buenos Aires, 2006. 
su pretensión de constituir un determinado tipo de sujeto infantil por medio de la educación y los consejos, sino además por la incidencia de su discurso en la subjetivación de los propios padres, es decir, la constitución de prácticas, discursos e identidades en torno a la maternidad y a la paternidad. ${ }^{8}$

La indagación de esta temática se entrecruza con los estudios sobre la construcción social de la infancia. Al respecto, en el ámbito local, durante los últimos años se ha producido una serie de trabajos provenientes del campo de la historia y las ciencias sociales, que parten de la idea de que la infancia no es una categoría descriptiva de una fase evolutiva natural, sino que se trata de una construcción singular, caracterizada por su contingencia, su historicidad y su relación con la política y la cultura. ${ }^{9}$ Estas investigaciones han abordado la relación que el discurso médico estableció con la infancia indagando diversos temas, tales como el proceso de individualización de la niñez por parte de la medicina infantil o las relaciones entre los médicos y la población, tanto en el marco de las políticas de protección materno-infantil, como a través de los consejos y prescripciones en revistas de difusión masiva y en otros dispositivos pedagógicos destinados a las madres. ${ }^{10}$ Estos y otros estudios consideran

8. DOS SANTOS LAMPRECHT, Claudia, "Conselhos às mães...", Op. Cit.

9. COSSE, Isabella, LLOBET, Valeria, VILLALTA, Carla y ZAPIOLA, María Carolina, "Introducción”, en COSSE, Isabella, LLOBET, Valeria, VILLALTA, Carla y ZAPIOLA, María Carolina (editoras) Infancias: politicas y saberes en Argentina y Brasil: siglos XIX y XX, Teseo, Buenos Aires, 2011, pp.11-28.

10. COLÁNGELO, María Adelaida, "El saber médico y la definición de una 'naturaleza infantil' entre fines del siglo XIX y comienzos del siglo XX en la Argentina”, en COSSE, Isabella, LLOBET, Valeria, VILLALTA, Carla y ZAPIOLA, María Carolina (editoras) Infancias: politi- a la maternidad como un fenómeno cultural que cambia a lo largo del tiempo y en el cual los aspectos materiales y simbólicos se entrecruzan y se influyen mutuamente. A su vez, han mostrado dos procesos fundamentales para analizar las representaciones y prácticas en torno al "binomio madre-hijo": la naturalización de la maternidad que intentó explicar una relación social por sus caracteres biológicos y la progresiva medicalización de la procreación y la crianza de los recién nacidos. ${ }^{11}$

A partir de estos aportes, el presente artículo se propone profundizar el estudio de las relaciones entre el saber médico y el conocimiento

cas y saberes..., Op. Cit., pp.101-121. BIERNAT, Carolina y RAMACCIOTTI, Karina, Crecer y multiplicarse: la politica sanitaria materno-infantil. Argentina 1900-1960, Biblos, Buenos Aires, 2013. BILLOROU, María José, "Madres y médicos en torno a la cuna. Ideas y prácticas sobre el cuidado infantil (Buenos Aires, 1930-1945)", en La Aljaba, Santa Rosa, 2007, V. XI, pp. 167-192. Disponible en: <http://www.scielo.org.ar/pdf/aljaba/v11/ v11a08.pdf> RUSTOYBURU, Cecilia, "Los consejos sobre crianza del Dr. Bonanfant: pediatría, psicoanálisis y escuela nueva (Buenos Aires, fines de la década de 1930)", en Temas y debates, Rosario, 2012, n 23, pp. 103124. Disponible en: <http://www.temasydebates.unr. edu.ar/index.php/tyd/article/view/31/31> BORINSKY, Marcela, "Todo reside en saber qué es un niño. Aportes para una historia de la divulgación de las prácticas de crianza en Argentina", en Anuario de investigaciones- Psicología UBA, Buenos Aires, 2005, V. XIII, pp. 117-126. Disponible en: <http://www.scielo.org.ar/pdf/anuinv/ v13/v13a43.pdf> BRIOLOTTI, Ana y BENÍTEZ, Sebastián, "Medicina, higiene mental y saber psi en la construcción de la maternidad y la infancia en la Argentina: un análisis a través de la revista MADRE Y NIÑO (1934-1935)", en Universitas Psychologica: Panamerican Journal of Psychology, Bogotá, 2014, V. XIII, n 5, pp. 17091719. Disponible en: <http://recursostic.javeriana.edu.co/ sitio/psychologica/sccs/articulo.php?id=7084\&PHPSESSID =d2593e255fb09a401 cf2a9b593193e77>; DARRÉ, Silvina, Maternidad y tecnologías de género, Katz Editores, Buenos Aires, 2013.

11. NARI, Marcela, Politicas de maternidad y maternalismo politico, Biblos, Buenos Aires, 2004. 
psicológico en la educación de los padres para el cuidado y la crianza de sus hijos, a través del análisis de dos manuales de puericultura relevantes en el ámbito local: El libro de las madres de Gregorio Aráoz Alfaro, cuya primera edición data de 1899 y La Salud del Hijo de Juan Garrahan, publicado en 1955. Ambos autores se destacaron por su trayectoria profesional y académica, $y$ fueron referentes importantes en el ámbito de la medicina infantil local. Aráoz Alfaro fue asimismo una figura pionera en la conformación de un extenso público lector por fuera del círculo de especialistas, hecho que puede inferirse a través de las sucesivas reediciones de su libro en 1908 y 1922. La obra de este pediatra y puericultor argentino estaba dirigida a las madres en general, pero particularmente a aquellas provenientes de los sectores sociales más pobres, que no siempre podían acceder rápida y fácilmente a la atención médica. Se trata de un texto sencillo y claro, con numerosas ilustraciones que pretendían clarificar las instrucciones del autor respecto de las medidas antisépticas necesarias para el parto, las maneras de vestir y abrigar al niño, la preparación de alimentos de manera higiénica, entre otras.

El libro de Juan Garrahan, por su parte, se inscribe en un campo de literatura de divulgación médica que, hacia mediados de los años cincuenta, se hallaba más consolidado. ${ }^{12}$

12. Durante la primera mitad del siglo XX, y en gran medida debido a la conformación de un público lector producto de la implementación de políticas educativas a gran escala, se desarrollaron numerosos emprendimientos destinados a la educación sanitaria de la población. En lo relativo a los temas de puericultura, los manuales, folletos y revistas fueron los principales canales de difusión, a los que se sumó más tarde la radio. $\mathrm{Al}$ respecto véase BRIOLOTTI, Ana y BENÍTEZ, Sebastián, "Medicina, higiene mental y saber psi...” Op. Cit. BRIOLOTTI, Ana, "Del 'pequeño salvaje' al individuo social: saber
Publicado en 1955 por la editorial El Ateneo y reeditado en 1963 por la editorial Médica Panamericana, La Salud del Hijo es un extenso texto de casi seiscientas páginas, dirigido no ya únicamente a la madre sino a ambos progenitores. Al igual que en el caso de Aráoz Alfaro, el libro de Garrahan tenía una aspiración eminentemente práctica y, en ese sentido, el estilo de escritura es llano y accesible, reforzado por ilustraciones, tablas y una sección final destinada a responder una serie de "preguntas imaginadas" que cualquier madre o padre podría realizar al médico. Asimismo, en su pretensión de satisfacer cierta curiosidad intelectual que atribuye al espíritu cultivado de los lectores, el autor intercala los consejos prácticos con información teórica y conceptual, brindando incluso un extenso apéndice de referencias bibliográficas.

Cada una de estas obras representa, como podrá apreciarse, un momento particular en lo relativo al modo cómo el discurso médico se dirigió a los padres, conceptualizó el desarrollo del niño y estableció problemas y soluciones en torno a su crianza. Así, el análisis de ambos textos permite dar cuenta tanto de las similitudes como de las diferencias en las formas de concebir el desarrollo infantil y los roles paternos. Esto implica a su vez atender

médico y desarrollo psicológico en la revista Hijo Mío..!", ponencia presentada en $V$ Congreso Internacional de Investigación y Práctica Profesional en Psicología, Universidad de Buenos Aires, Buenos Aires, 2013, pp. 29-32. Disponible en: <http://www.aacademica.org/000-054/117. pdf> [Consulta: 27 octubre 2014]. BRIOLOTTI, Ana, "La voz de la ciencia educando a los padres: el desarrollo psicológico del niño en las conferencias radiales dirigidas a la comunidad platense (1942-1947)", en VIGUERA, Ariel (coordinador) Historias de la psicología y el psicoanálisis en La Plata (1946-1990), EDULP, La Plata, 2015. RUSTOYBURU, Cecilia, "Los consejos sobre crianza del Dr. Bonanfant...”, Op. Cit. BORINSKY, Marcela, “Todo reside en saber...", Op. Cit. 
a ciertas particularidades relativas al contexto de producción de cada obra, que incidieron en la caracterización de la crianza y de la propia puericultura.

A lo largo del artículo se revisarán, en primer lugar, algunos aspectos relativos al contexto de surgimiento de la educación de los padres en el mundo occidental y sus particularidades en la Argentina. En segundo lugar, se indagará la obra de Aráoz Alfaro, recorriendo ciertos temas y problemas que la atraviesan y que permiten dar cuenta de los propósitos que perseguía la iniciativa de educación de las madres en el contexto de consolidación de la Argentina como Estado-nación. Por último, el análisis del manual de Juan Garrahan permitirá mostrar ciertos cambios significativos que tuvieron lugar hacia mediados del siglo XX y que implicaron una reconfiguración en las formas de concebir el desarrollo infantil y, por ende, los deberes parentales vinculados con la crianza.

\section{Educando a los padres para la salud de sus hijos: la medicina al servicio de la nación}

En el mundo occidental, las acciones educativas vinculadas con el cuidado de los niños fueron impulsadas en el contexto de las transformaciones que sufrió la maternidad entre fines del siglo XIX y la primera mitad del siglo XX, período que coincide con la denominada "glorificación de la maternidad" ${ }^{13}$ En el marco de este proceso, las mujeres fueron mantenidas por fuera del ámbito público en

13. KNIBIEHLER, Yvonne, Historia de las madres $y$ de la maternidad en Occidente, Nueva Visión, Buenos Aires, 2001. virtud de su deber "natural" de educar futuros ciudadanos sanos y fuertes. En un contexto marcado por los discursos sobre la cantidad y la calidad de la población como factor decisivo para el desarrollo de las naciones y por el ascenso progresivo de la higiene y la medicina social, la divulgación de conocimientos científicos en el marco de la educación de las madres cobró gran relevancia en la prevención de enfermedades y anormalidades infantiles. De esta manera, la tarea del médico se extendió más allá de la consulta personal y se vinculó con la creciente intervención del Estado en la gestión racional y eficiente del capital humano de la nación. De acuerdo con la cosmovisión positivista hegemónica, la ciencia se consideraba el instrumento privilegiado para lograr un orden social racional y la nación era pensada como un cuerpo, un organismo al que debía cuidarse y velar por la salud física y moral de sus pobladores. ${ }^{14}$

En la Argentina, las iniciativas médicas vinculadas con la asistencia materno-infantil y la educación de las mujeres en lo relativo al cuidado de sus hijos surgieron a fines del siglo XIX en torno a dos problemas fundamentales: las altas tasas de mortalidad infantil y el elevado número de niños en condiciones de abandono. Si bien se trataba de fenómenos de más larga data, desde la década de 1880 se agravaron en el marco de un panorama de acelerada industrialización y modernización dado por la incorporación de la Argentina a los mercados mundiales a través de la actividad agroexportadora y por la gran afluencia inmigratoria proveniente de Europa. Esto dio impulso a toda una serie de estrategias que,

14. MURILLO, Susana, La ciencia aplicada a políticas sanitarias en Argentina y su relación con la escuela de medicina de la Universidad de Buenos Aires (1869-1905), Tesis de Maestría, Universidad de Buenos Aires, 2001. 
bajo la forma de educación moral y médica, reformas sociales y políticas sanitarias se propusieron evitar el abandono y el descuido de los niños, al tiempo que participaron en la construcción del concepto de madre. ${ }^{15}$

En cuanto al surgimiento de las iniciativas de divulgación, tal como ha sido señalado para el caso de Brasil a principios del siglo XX, el saber médico se autoproclamó indispensable para vigilar y gobernar las conductas de los adultos, fundamentalmente de las madres, a fin de asegurar la supervivencia de los niños y mejorar sus condiciones de salud. ${ }^{16}$ En la Argentina, este hecho coincidió con el proceso de profesionalización de la medicina, que se inició en el último tercio del siglo XIX e implicó la legitimación de su saber y la obtención del monopolio de la atención a la salud. Este proceso se dio de forma paralela a la consolidación del Estado, de modo tal que el saber y las prácticas médicas acompañaron la lógica de su crecimiento y desarrollo. En este marco y adoptando el modelo francés, la figura del médico se vinculó de manera estrecha con la higiene, centrada en la prevención y en la intervención en el ámbito público. ${ }^{17}$

\section{BIERNAT, Carolina y RAMACCIOTTI, Karina, Crecer y multiplicarse..., Op. Cit. GUY, Donna, "Niños abandonados en Buenos Aires (1880-1914) y el desarrollo del concepto de la madre", en FLETCHER, Lea, Mujeres y cultura en la Argentina del siglo XIX, Feminaria Editora, Buenos Aires, 1994.}

16. DE MOURA, Marina, "Saúde e educação na revista Infância”, en Cadernos de História da Ciência. Instituto Butantan, San Pablo, 2010, V. VI, No 2, pp. 27-43. Disponible en: <http://teste.pc2.com.br/butantan/ arquivos/31/PDF/v6n2a02.pdf >

17. GONZÁLEZ LEANDRI, Ricardo, "Itinerarios de la profesión médica y sus saberes de Estado. Buenos Aires, 1850-1910”, en PLOTKIN, Mariano y ZIMMERMANN, Eduardo (compiladores) Los saberes del Estado, Edhasa, Buenos Aires, 2012.
Fue así que la medicina se articuló con las políticas públicas destinadas a controlar los fenómenos asociados a la "cuestión social", vale decir, los efectos de la industrialización y de la urbanización que, a los ojos de las élites políticas e intelectuales, amenazaban la estabilidad del orden social. ${ }^{18}$ En este marco, el discurso y las prácticas médicas procuraron intervenir a nivel de las conductas de la población. Una de las estrategias adoptadas para tal fin fue precisamente la divulgación de conocimientos a través de la prensa escrita y los libros.

Las fuentes analizadas en este artículo muestran que a lo largo de las décadas se mantiene incólume la idea de que la intervención del médico se funda en la solidez de su saber, acreditada por una formación científica rigurosa que lo convierte en el único conocimiento certero y fiable. Así, a fines del siglo XIX Aráoz Alfaro situaba a la figura del médico como "el mentor autorizado de la familia y de la sociedad" ${ }^{19}$, mientras que años más tarde Garrahan sostendría que el deber del puericultor de actuar como consejero en la intimidad de la familia le permitía realizar una importante obra social, al tiempo que

GONZÁLEZ LEANDRI, Ricardo, "Notas acerca de la profesionalización médica en Buenos Aires durante la segunda mitad del siglo XIX", en SURIANO, Juan (compilador) La cuestión social en Argentina, 1870-1943, La Colmena, Buenos Aires, 2004.

18. SURIANO, Juan, "Introducción: una aproximación a la definición de la cuestión social en Argentina”, en SURIANO, Juan (compilador) La cuestión social en Argentina, 1870-1943, La Colmena, Buenos Aires, 2004.

19. ARÁOZ ALFARO, Gregorio, El libro de las madres. Pequeño tratado práctico de higiene del niño con indicaciones sobre el embarazo, parto y tratamiento de los accidentes, Librería científica de Agustín Etchepareborda, Buenos Aires, 1899, p. XV. 
ennoblecía su profesión. ${ }^{20}$

Si bien dista de ser exhaustivo, el panorama aquí esbozado pone de manifiesto algunas ideas fundamentales en torno a las iniciativas médicas vinculadas con la educación de los padres, que permitirán en lo que sigue iluminar ciertas continuidades y rupturas por medio del análisis de dos obras, una de ellas publicada en los albores del siglo XX y la otra a mediados de la década de 1950.

\section{El libro de las madres de Aráoz Alfaro: instruir para el progreso de la nación}

En 1899, año en que se publica El libro de las madres, Gregorio Aráoz Alfaro daba sus primeros pasos en una carrera profesional destacada que culminó en 1955, con su muerte a los 85 años de edad. Oriundo de la provincia de Tucumán, en 1892 se había graduado de médico con Diploma de Honor de la Universidad de Buenos Aires. Durante un tiempo fue Director honorario de los Consultorios del Patronato de la Infancia, institución fundada en 1892 con el fin de proteger a los niños contra el abandono, la enfermedad, los malos tratos y la inmoralidad. ${ }^{21}$ Asimismo, desde 1892, se desempeñaba como Secretario de la Dirección General de la Asistencia Pública y Administración sanitaria, bajo la dirección del higienista Emilio Coni. Era además médico de niños del Hospital San

20. GARRAHAN, Juan Pedro, La Salud del Hijo.

Puericultura, El Ateneo, Buenos Aires, 1955.

21. COWEN, Miguel Pablo, "Infancia, abandono y padres en el siglo XIX porteño", en Anuario del Instituto de Historia Argentina, La Plata, 2004, No 4, pp. 75-99.

Disponible en: <http://www.fuentesmemoria.fahce.unlp. edu.ar/art_revistas/pr.3249/pr.3249.pdf> [Consulta: 9 de marzo 2014]

46
Roque y profesor del Colegio Nacional de Buenos Aires.

La primera edición de El libro de las madres data, como se mencionó, de 1899 y tuvo dos reediciones, en 1908 y en 1922. Esta última incluyó un breve capítulo referido a la eugenesia, disciplina que Aráoz Alfaro pretendía difundir motivado por el anhelo de forjar el país con una población sana y vigorosa. ${ }^{22}$ En el prefacio de la primera edición, el autor presentaba su libro como una pequeña obra de vulgarización que indirectamente pretendía beneficiar al médico evitándole la pérdida de tiempo en la reiteración de explicaciones. Pero el libro estaba destinado fundamentalmente a las madres de familias modestas dado que, según se afirmaba, era dificultoso para este sector de la población recurrir al médico. Si bien $E l$ libro de las madres se publicó en un momento en el cual la medicina comenzaba a lograr el reconocimiento y la legitimación por parte de la sociedad, es preciso tener en cuenta que a lo largo del último tercio del siglo XIX la lectura de manuales de medicina casera, la automedicación y los consejos de familiares o vecinos eran los modos más frecuentes de enfrentar los malestares. ${ }^{23}$ Con respecto al hábito de seguir el consejo de los legos, Aráoz Alfaro lo descalificaba afirmando que las recomendaciones no expertas, legitimadas por la experiencia pero no por ello menos

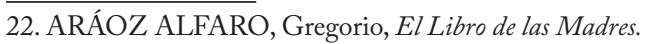
Manual práctico de higiene del niño, con indicaciones sobre el embarazo, parto, y tratamiento de los accidentes. Nueva edición reformada, Cabaut y Cía. Editores, Buenos Aires, 1922.

23. ARMUS, Diego y BELMARTINO, Susana, "Enfermedades, médicos y cultura higiénica", en CATTARUZZA, Alejandro (director) Crisis económica, avance del Estado e incertidumbre política (1930-1943), Colección Nueva Historia Argentina, T. VII, Sudamericana, Buenos Aires, 2001, 283-329. 
ignorantes, eran responsables de los graves errores en la crianza cometidos por las madres. De igual modo que en otros contextos, el discurso de la puericultura argentina delimitó su especificidad y su rigor científico contraponiéndose a los saberes populares, considerados erróneos y perjudiciales para el niño. ${ }^{24}$

El libro de las madres era ante todo una guía para prevenir enfermedades tanto de la mujer embarazada y puérpera como del niño. Desarrollaba en extenso las medidas antisépticas necesarias para evitar la muerte por fiebre puerperal, enfermedad que seguía haciendo estragos sobre todo en las provincias, donde las familias e incluso las parteras no contaban con información respecto de la antisepsia en el parto. En lo que respecta al niño, el manual se proponía contribuir a la higiene de la infancia, etapa de la vida en la que era primordial recibir cuidados adecuados -sobre todo en lo relativo a la alimentaciónpara prevenir enfermedades. En la pluma del autor, estos propósitos inspirados en el higienismo se conjugaban con un sentimiento patriótico que veía en la divulgación científica una vía privilegiada para el progreso de la sociedad y del país:

Vulgarizar estos preceptos, tratar de introducirlos en los hogares argentinos con la autoridad que puedan dar muchos

24. DE LUNA FREIRE, Maria Martha, "'Ser mãe é uma ciência': mulheres, médicos e a construção da maternidade científica na década de 1920", en História, Ciências, Saúde-Manguinhos, Rio de Janeiro, 2008, V. 15, suplemento, pp. 153-171. Disponible en: <http:// www.scielo.br/pdf/hcsm/v15s0/08.pdf> DE MOURA, Marina, “Saúde e educação...”, Op. Cit.; DOS SANTOS LAMPRECHT, Claudia, "Conselhos às mães...", Op. Cit. LOREDO NARCIANDI, José Carlos, "La construcción de niños y niñas...", Op. Cit. años de dedicación á la medicina infantil, es, á mi juicio, una obra de humildad y de patriotismo... Estoy convencido de que si consigo expresar en este libro, con bastante claridad y exactitud, las ideas y los preceptos racionales que deben presidir la crianza del niño, habré realizado una obra mucho más provechosa para la sociedad y para el país que con todos mis trabajos propiamente científicos. ${ }^{25}$

Es en este sentido, afirmamos que la preocupación primordial de los médicos puericultores en los inicios del siglo XX radicaba en el bienestar infantil y, a través suyo, en la prosperidad de la nación. ${ }^{26}$

Si bien el texto se ocupaba de la higiene del vestido y de la habitación, del aseo corporal y de la educación física e intelectual, desarrollaba en mayor profundidad los aspectos relativos a la alimentación, por considerarla de importancia capital para la infancia. Aráoz Alfaro destinaba buena parte del texto a argumentar en favor de la llamada "lactancia natural". Las recomendaciones de nuestro autor se alineaban con una serie de textos que, ya desde el último tercio del siglo XIX, trataban de mostrar las ventajas de la alimentación a pecho, a la que muchas mujeres se resistían, entre otras razones, a causa del temor a perder sus encantos físicos. ${ }^{27}$

Eran variadas las razones por las cuales la alimentación a pecho era preferible a la lactancia "artificial" con leche animal y otros

25. ARÁOZ ALFARO, Gregorio, El libro de las madres.., Op. Cit., p. IX.

26. LOREDO NARCIANDI, José Carlos, “La construcción de niños y niñas...”, Op. Cit.

27. ESCARDÓ, Florencio, "Las tesis sobre lactancia", en Archivos Argentinos de Pediatria, Buenos Aires, 1943, V. XIV, No 6, pp. 448-455. 
preparados. En primer lugar, su valor venía dado por el bajo índice de enfermedades y trastornos digestivos entre los niños alimentados a pecho. La composición de la leche de mujer era la que mejor se adecuaba a las capacidades digestivas del niño, además de ser casi nulo el riesgo de portar microbios responsables de enfermedades infecciosas. Asimismo, el desarrollo evidenciaba diferencias, ya que en los niños alimentados artificialmente el peso no parecía aumentar con la misma regularidad, la fontanela tardaba más en cerrarse y se retrasaban la dentición, el logro de la posición erguida y la marcha. En segundo lugar, y en conexión con lo anterior, la lactancia natural entrañaba ventajas macro y microsociales: por un lado, el niño saludable generaba menos gastos y menos sufrimientos a su familia. Por otro lado, la sociedad se beneficiaba conservando un mayor número de individuos sanos y fuertes. A estos argumentos en favor de la crianza con leche humana se añadía otra premisa: preferentemente, el niño debía alimentarse con leche de su madre. Aquella parecía ser la más adecuada, dado que provenía del organismo en el cual el niño se había gestado y constituía una suerte de "sangre blanca" que prolongaba el lazo vital entre la mujer y su hijo luego del alumbramiento. Este tipo de aseveraciones eran parte de una retórica centrada en el binomio madre-hijo, unidad funcional de base biológica que sirvió de fundamento a los preceptos de crianza difundidos por los médicos. Desde esta óptica biologicista, el amamantamiento era visto como una obligación que la naturaleza imponía a las madres, cuya desobediencia -no pocas veces tildada de "inmoral"- podía acarrear graves consecuencias para la salud y la supervivencia del niño. El discurso sobre la lactancia materna fue así parte importante de los procesos de medicalización de la procreación y la crianza y de naturalización de la maternidad, que, apelando a fundamentos biológicos y morales, contribuyeron a la consolidación de la idea de una mutua dependencia entre la madre y su hijo. ${ }^{28}$

Es de destacar que el fundamento último en la promoción de la lactancia materna era la supervivencia del niño, cuya robustez y salud física eran las metas principales de la crianza. En este punto cabe recordar que, en los albores del siglo XX, la mortalidad infantil seguía siendo una de las preocupaciones centrales de cara al desarrollo y al progreso de la nación. En el caso de Buenos Aires, la tasa de mortalidad de la primera infancia había trepado a 404.7 por mil nacimientos en el quinquenio 18651869. Y si bien desde 1870 se evidenciaba un descenso considerable, las sucesivas epidemias que afectaron a la población porteña entre 1869 y 1900 produjeron fluctuaciones en los niveles de mortalidad, elevando el número de casos de defunción por enfermedades infecciosas. ${ }^{29}$ En este escenario, es lógico suponer que las iniciativas médicas vinculadas con la educación para el cuidado de los hijos hicieran énfasis en aquellas medidas que podían asegurar la supervivencia del niño.

El tratamiento de las pautas relativas a la alimentación, al sueño y al resto de las necesidades fisiológicas se destaca por el énfasis de Aráoz Alfaro en la necesidad de ejecutar las prescripciones a un ritmo inquebrantable,

28. NARI, Marcela, Políticas de maternidad..., Op. Cit.

29. MAZZEO, Victoria, "La mortalidad de la primera infancia en la Ciudad de Buenos Aires en el periodo 1860-2002", en Papeles de Población, México, 2007, V. XIII, No 53, pp. 241-273. Disponible en: $<$ http://rppoblacion.uaemex.mx/pp/index.php/ papelesdepoblacion/article/view/396/384> 
respetando los horarios estipulados por el médico. Esta insistencia en la necesidad de control pone de manifiesto el ideal de un sujeto disciplinado y autogobernado que, con diferentes matices, parece caracterizar a la puericultura a lo largo de la primera mitad del siglo $\mathrm{XX} .{ }^{30}$ En el marco de este planteo, una manifestación habitual como el llanto del lactante era interpretada en función de la necesidad de mantener inalterado el orden y la secuencia de los cuidados:

Los padres tienen, pues, que hacerse sordos á los gritos del niño y aunque les duela, es preciso no dejarles adquirir malas costumbres, como sucede cuando se les da el pecho cada rato, ó cuando se les levanta y pasea momento por momento. El niño, tomado el pecho ó cambiado las ropas, debe ser puesto en su cuna y dejado allí, sin mecerlo ni hamacarlo. Si llora y se está seguro de que no es por frío ó por mal arreglo de las ropas, ó por un alfiler que lo pincha ú otra causa por el estilo, hay que dejarlo llorar: un momento después, calla y se duerme tranquilamente.

Si se decide uno á sufrir los gritos durante 10 ó 15 días, se puede estar seguro de no tener en la casa un pequeño tiranuelo caprichoso y molesto. ${ }^{31}$

Si bien no es posible precisar cuáles eran las referencias de nuestro autor en los temas de psicología infantil, puede afirmarse que la imagen del niño como un potencial tirano -dominante en el campo médico de las primeras décadas del siglo pasado- era el reflejo de una concepción de corte naturalista y evolucionista

30. LOREDO NARCIANDI, José Carlos, "La construcción de niños y niñas...”, Op. Cit.

31. ARÁOZ ALFARO, Gregorio, El libro de las madres..., Op. Cit., p. 64. que caracterizó a la psicología local en los comienzos del siglo $\mathrm{XX}^{32}$ De acuerdo con este modelo biológico del desarrollo psíquico, cada individuo recapitulaba durante la infancia las fases de la evolución de la especie. Esto justificaba el carácter apasionado y caprichoso del niño, que reeditaba en sus actitudes ciertas tendencias ancestrales, producto de la herencia filogenética. ${ }^{33}$ De allí que la voluntad del niño debiera plegarse a la de sus padres y maestros, que serían los encargados de educar su espíritu. Sin dejar de lado la necesidad de brindar cariño al hijo, es notable la insistencia del autor en la idea de que la educación es ante todo una obra de autoridad y de respeto.

Además de dar cuenta de los tiempos y ritmos estipulados para el desarrollo de los sentidos y del lenguaje, el autor destinó un segmento de la obra a la educación moral e intelectual. Con respecto a esta última, se indicaba no anteponer la instrucción propiamente dicha a la higiene corporal y a la educación física: los padres debían saber que "apurar fuera de medida" la inteligencia del niño nunca podía ser más importante que procurar su salud, su

32. La psicología evolutiva del niño surgida en el marco de la perspectiva evolucionista produjo sus primeros textos en el último cuarto del siglo XIX, a partir de autores como Taine, Darwin y Preyer. Entre otras referencias que tuvieron recepción en la Argentina, podrían mencionarse los trabajos de Baldwin, Hall, Compayré y Claparède, entre otros.

33. RÍOS, Julio César y TALAK, Ana María, "La niñez en los espacios urbanos", en DEVOTO Fernando y MADERO, Marta (editores) Historia de la vida privada en la Argentina, La Argentina plural (1870-1930), T.

II:, Taurus, Buenos Aires, 1999. TALAK, Ana María,

"E1 desarrollo psicológico entre la naturaleza, la cultura y la política (1900-1920)", en GARCÍA, Luciano, MACCHIOLI, Florencia y TALAK, Ana María Psicología, niño y familia en la Argentina 1900-1970: perspectivas históricas y cruces disciplinares, Biblos, Buenos Aires, 2014. 
crecimiento y su fortaleza. ${ }^{34}$ En esa dirección, Aráoz Alfaro señalaba que los niños precoces no llegaban muy lejos cuando crecían y que la inteligencia prodigiosa solía ser el resultado de cerebros degenerados y enfermos.

En lo relativo a la educación moral, y del mismo modo que en las demás esferas del desarrollo, el autor destacaba el rol primordial de la madre. El libro comenzaba con dos epígrafes, uno de los cuales afirmaba "El futuro de un niño es siempre obra de una madre". Bajo esta premisa, las madres eran las responsables de modelar esa blanda masa cuya organización física y moral parecía depender del impulso recibido durante los primeros años de vida. $\mathrm{Al}$ respecto cabe señalar que si bien la concepción de desarrollo psicológico dominante en esos años era determinista y biologicista, no por ello desestimaba el rol del ambiente como corrector o facilitador de ciertas tendencias. ${ }^{35}$

El rol estipulado para la madre en los primeros años de vida del hijo estaba basado en un argumento implícito que equiparaba femineidad y maternidad, y que fue la base del proceso de "maternalización" de las mujeres en Occidente. De aquí se desprendió la idea de maternidad no sólo como posibilidad sino como destino irrevocable. ${ }^{36}$ Ahora bien, la madre a la que hacía referencia el autor se caracterizaba por su abnegación en pos de la crianza de sus hijos: era la mujer "realmente afectuosa" ${ }^{37}$, es decir, aquella que dejaba de

34. ARÁOZ ALFARO, Gregorio, El libro de las madres.., Op. Cit., p. 276.

35. TALAK, Ana María, "El desarrollo psicológico...", Op. Cit.

36. NARI, Marcela, Politicas de maternidad..., Op. Cit.

37. ARÁOZ ALFARO, Gregorio, El libro de las madres..., Op. Cit., p. 280. lado los paseos y diversiones por cuidar de su progenie. Para esa madre, la tarea de formar el alma del niño no era otra que la de educar el sentimiento e inspirar en sus hijos el amor al trabajo, el entusiasmo y el sacrificio. En definitiva, el más noble de los deberes maternos consistía en dar a la sociedad hombres afectuosos, caritativos, honestos, útiles, de carácter firme, "tan alejados de la irritabilidad exagerada de un sentimentalismo infecundo como de la cínica dureza del egoísmo". ${ }^{38}$

A continuación intentaremos poner de manifiesto las similitudes y las diferencias entre este momento de la educación de los padres y el que configura la obra de Juan Garrahan, publicada unas décadas más tarde. Esta comparación permitirá reflexionar sobre las modificaciones en torno al desarrollo infantil y la crianza, y el modo como dichos cambios se reflejaron en la forma de conceptualizar la tarea del puericultor.

\section{Juan Garrahan y la "nueva puericultura": la libertad dentro del orden}

A mediados de la década de 1950, Juan Garrahan (1893-1965) era un pediatra de reconocida trayectoria. Había obtenido su título de médico en 1915 con Diploma de Honor de la Universidad de Buenos Aires. Desde mediados de la década de 1920 hasta los años cuarenta trabajó en el Instituto de Maternidad (Prof. Peralta Ramos) del Hospital Bernardino Rivadavia. En 1942, había obtenido la titularidad en la cátedra de Clínica Pediátrica y Puericultura y la dirección del Instituto de Pediatría del Hospital de Clínicas de la Universidad de Buenos Aires.

38. Ídem, p. 282 
Entre 1953 y 1955, se mantuvo alejado de la universidad por motivos políticos, pero continuó desarrollando una profusa actividad en el ámbito privado. A fines de 1955, luego del golpe militar que derrocó al segundo gobierno de Juan Domingo Perón, sería reincorporado a sus tareas en la universidad. ${ }^{39}$

En su libro, Garrahan se identificaba con las nuevas corrientes de la puericultura, disciplina que hasta el momento había centrado su interés en el estudio de las diferenciaciones anatómicas $\mathrm{y}$ funcionales del niño y sus exigencias de alimentación y cuidado, impartiendo una serie de prescripciones rígidas, fundadas en mediciones y cronologías del desarrollo. ${ }^{40}$ A juicio del autor, se trataba de un esquema demasiado simple que se ocupaba del niño en un sentido abstracto, dejando de lado la comprensión de su individualidad familiar y racial y de su maduración psíquica:

hasta hace poco, el "pobre niño", y sobre todo el "pobre lactante", era tratado y considerado como si estuviera "desprovisto de alma”... Por fortuna, en los años últimos se ha operado una reacción muy beneficiosa en materia de crianza y educación del niño: se valora más actualmente el significado del afecto, contemplándose también el "alma del niño", y preconizándose en consecuencia, la necesidad de orientar y

39. GARRAHAN, Juan Pedro, "La Universidad argentina y el momento actual", en Archivos de Pediatría del Uruguay, Montevideo, 1945, V. XVI, No 10, pp. 65354; GARRAHAN, Juan Pedro, Antecedentes, Titulos y Trabajos de Pediatría y Puericultura, Universidad de Buenos Aires - Facultad de Ciencias Médicas, Buenos Aires, 1956; GARRAHAN, Juan Pedro. "Tres años de labor pediátrica extrauniversitaria”, en La Semana Médica, Buenos Aires, 1956, V. LXIII, No 3241, pp. 251-54.

40. GARRAHAN, Juan Pedro, "La nueva Puericultura”, Archivos de Pediatría del Uruguay, Montevideo, 1955, V. XXVI, No 1, pp. 128-134. moldear armónicamente desde los primeros meses de vida el desarrollo físico, emotivo e intelectual. $^{41}$

En rigor de verdad, ya desde los años cuarenta, Garrahan reflexionaba acerca de este tópico. En 1942, en el discurso pronunciado en ocasión de obtener la titularidad de la cátedra de Clínica Pediátrica y Puericultura, el autor sostenía que los pediatras y puericultores debían ser médicos del cuerpo y del alma, dada la importancia del factor psíquico en la génesis de ciertos procesos mórbidos de la niñez. ${ }^{42} \mathrm{El}$ enfoque de la "nueva puericultura" proponía entonces adaptar los consejos y las pautas de crianza a la particularidad psico-física de cada niño y sobre todo a las variables emocionales. De lo contrario se corría el riesgo de frustrar el despliegue de la singularidad del niño y desviar su personalidad, desencadenando así ansiedad o trastornos de conducta.

El texto de Garrahan se orientaba en dirección a facilitar el manejo diario de los niños. Antes que de la noción generalizada y en cierto modo abstracta de "niño", el autor prefería ocuparse del "hijo" en tanto realidad que concretaba el deseo de los progenitores y se ligaba al más refinado de los sentimientos. Si el libro de Aráoz Alfaro se dirigía a la madre, los interlocutores de la obra de Garrahan eran ambos padres, de quienes se esperaba un interés por dirigir sabiamente la crianza y la educación de sus hijos y por cuidar con eficiencia su salud. Al igual que en Aráoz Alfaro, el espíritu

41. GARRAHAN, Juan Pedro, La Salud del Hijo..., Op. Cit., p. 557.

42. GARRAHAN, Juan Pedro, "Facultad de Ciencias Médicas de Buenos Aires. Cátedra de Clínica Pediátrica y Puericultura. Conferencia inaugural $\left(1^{\circ}\right.$ de Octubre de 1942)", en Anales de la Sociedad de Puericultura de Buenos Aires, Buenos Aires, 1942, V. VIII, No 4, pp. 368-84. 
de la obra de Garrahan era informar, enseñar y evacuar dudas. Sin embargo, el texto iba más allá del mero interés práctico: pretendía asimismo satisfacer las exigencias emotivas de los padres, quienes además de directivas concretas requerían apoyo moral para resolver los múltiples problemas que les planteaba la crianza. En ese sentido, Garrahan insistía en la necesidad de resguardar el equilibrio emocional de los padres y cuidadores, dada su ligazón con el bienestar del niño:

...un factor muy pernicioso para la crianza del niño... para la normal formación de hábitos, para el estímulo eficiente de su desarrollo fisiológico (cosas que repercuten sobre su salud), es el estado de ánimo de los padres en relación a sus hijos: el vivir descontentos de las condiciones físicas y funcionales de los mismos, y de su conducta, el exteriorizar ante ellos dicho estado de ánimo, el criarlos y educarlos bajo el dominio de una tal tensión emocional, es causante de muchos males para los pobres hijos. ${ }^{43}$

El núcleo de la renovación propuesta por Garrahan retomaba las ideas de ciertas figuras del campo médico-psicológico anglosajón, tales como el psicólogo y pediatra Arnold Gesell, la psicoanalista Susan Isaacs y la psiquiatra Margaret Ribble. Asimismo, se destacan las menciones al libro Baby and Child Care, del pediatra Benjamin Spock, quien, durante la Segunda Posguerra se convirtió en el nuevo gurú de la crianza en Estados Unidos, desplazando a Gesell de dicho lugar. En su obra -considerada uno de los mayores bestsellers de todos los tiempos- Spock proponía una nueva forma de encarar la crianza del niño, fundada en el afecto, la comprensión, el

43. GARRAHAN, Juan Pedro, La Salud del Hijo..., Op. Cit., p. 49. respeto por la individualidad y la confianza de los padres en su propia intuición y en sus capacidades. ${ }^{44}$

Basándose en estos aportes, Garrahan sostenía que en la crianza y educación del niño era preciso contemplar su particularidad, que venía dada por la personalidad, las aptitudes y la singularidad de los ritmos madurativos. Para los padres, esto implicaba conducirse con comprensión y elasticidad, adoptando un criterio amplio al interpretar los parámetros de normalidad. La estrictez, las ansiedades desmedidas y la falta de serenidad en el juicio de los padres parecían constituir el punto de partida de numerosos trastornos infantiles $y$, a nivel del desarrollo neuropsíquico, de retrasos en la marcha,la palabra, el control de esfínteres y el desarrollo intelectual. Un ejemplo ilustrativo a este respecto es el de la alimentación, tema que, al igual que en la obra de Aráoz Alfaro, recibía un tratamiento pormenorizado. Garrahan reconocía la importancia de la teoría de la nutrición, que pautaba la cantidad y la calidad del alimento necesarias para el ser humano. Pero, nuevamente aquí, se debían tener en cuenta las variantes individuales y los factores psíquicos que incidían en la determinación del apetito. En este punto era fundamental evitar las influencias psicológicas nocivas, resultantes del empeño de los padres en satisfacer las exigencias teóricas de la nutrición. Así, en lo respectivo al horario y a diferencia de Aráoz Alfaro, el autor descartaba el método de imponerlo desde el nacimiento y respetarlo rigurosamente con el objeto de formar en el niño hábitos acordes a la vida

44. SULMAN, Michael, "The humanization of the American child: Benjamin Spock as a popularizer of psychoanalytic thought", en Journal of the History of the Behavioral Sciences, New Hampshire, 1973, V. IX, No 3, pp. 258-265. 
familiar y social. Si bien en algunos casos este método parecía ser exitoso, podía conducir, según Garrahan, a la inapetencia rebelde, que en aquellos años constituía el motivo de consulta pediátrica más frecuente. ${ }^{45}$ Frente a este panorama, parecía más razonable un nuevo procedimiento de alimentación basado en el apetito del lactante, que respondiera a su demanda. Pero la adopción de este método, propuesto, entre otros, por Gesell, no implicaba dar de mamar al niño cada vez que lloraba, sino que requería de la capacidad de la madre para moderar las demandas del niño, desarrollando habilidades que le permitiesen reconocer el llanto por hambre.

En el texto, Garrahan reiteraba las ideas de Aráoz Alfaro en relación con la higiene, la pureza y la adecuación nutricional de la alimentación a pecho, aunque incorporando una dimensión ausente en El libro de las madres: la cualidad del vínculo afectivo que se establecía entre la madre y el hijo a través del amamantamiento. El autor destacaba la importancia psicológica de dicho vínculo, señalando su incidencia en el grado de equilibrio de las conductas futuras del niño y de sus reacciones emocionales. La lactancia materna seguía siendo valorada, pero, a diferencia del primer momento analizado, el derecho del lactante de recibir leche de su madre estaba motivado no tanto por la preocupación acerca de las posibles consecuencias negativas de la

45. BRIOLOTTI, Ana, "El problema de la inapetencia de origen psíquico en la primera infancia. Un aporte para el análisis de las relaciones entre pediatría, psicología y psicoanálisis en la Argentina (1936-1956)", ponencia presentada en 4tas Jornadas de Estudios sobre la Infancia: Lo público en lo privado y lo privado en lo público, Universidad Nacional de General Sarmiento, Buenos Aires, 2015, pp. 52-72. Disponible en: <https://www. aacademica.org/4jornadasinfancia/16.pdf> [Consulta: 12 junio 2015]. lactancia artificial para el organismo infantil, sino por la convicción de que el desarrollo físico y espiritual del niño dependían del pecho y del afecto de su madre.

Como puede apreciarse, si bien La Salud del Hijo recorre ciertos temas clásicos en puericultura, que estaban ya presentes en El libro de las madres, la mirada de Garrahan se detiene en los pormenores del desarrollo emocional, por considerarlo de suma importancia para juzgar la conducta del niño y orientar su educación:

el cuidado inteligente del hijo no consiste sólo en alimentarlo, protegerlo de los contagios y peligros, enseñarle hábitos de persona educada y darle instrucción. Es menester que [los padres] tengan presente, además, que el carácter, la personalidad emocional, repercuten en la salud y pueden llegar a ser factor de buen éxito o de fracaso en el futuro del niño, y que el preocuparse en lograr que el hijo llegue a ser equilibrado emocionalmente, es trabajar en favor de su futura felicidad. ${ }^{46}$

A diferencia de la insistencia de Aráoz Alfaro en la importancia de la robustez y la salud física del niño, Garrahan hacía especial hincapié en la necesidad de atender a las vicisitudes de su desenvolvimiento emocional, poniendo así en primer plano la capacidad de los padres para comprenderlas y conducirlas adecuadamente.

E1 recorrido realizado hasta aquí permite constatar que, a lo largo de las décadas transcurridas entre las obras analizadas, la tarea de criar niños parece haberse complejizado, hecho que se reflejó en las iniciativas médicas en torno a la educación de los padres. En este segundo momento la

46. GARRAHAN, Juan Pedro, La Salud del Hijo..., Op. Cit., p. 27. 
dimensión afectiva comenzó a posicionarse en el centro de la escena, relegando a un segundo plano los aspectos relativos a la supervivencia y al desarrollo físico y propiciando un diálogo más fluido entre la medicina y los saberes psicológicos. Esta modificación al interior de la puericultura formó parte de una serie de transformaciones en las concepciones de crianza, en cuyo marco la calidad de los vínculos establecidos entre los miembros de la familia se convirtió en una suerte de grilla de lectura para comprender el desarrollo psicofísico del niño e intervenir en su curso. Así, hacia los años cincuenta, el paradigma de maternidad y crianza sufriría una reconfiguración inscripta en un proceso más amplio que tuvo lugar en el mundo occidental luego de la Segunda Guerra Mundial. Este proceso implicó una nueva forma de concebir los problemas infantiles y, por ende, el desarrollo "normal". Si hasta entonces predominaba la idea de que el alma del niño era el resultado de predisposiciones heredadas y de una serie de hábitos adquiridos por su capacidad de imitar los modelos ofrecidos por el entorno, en este momento la mirada se posó sobre la familia entendida como un conjunto de relaciones. Desde esta perspectiva "familiarista", y aun cuando no se dejaran de lado por completo las concepciones previas, el nivel de normalidad del niño comenzó a ponderarse en función del grado de equilibrio emocional de los padres y de la calidad de los vínculos establecidos con sus hijos, de suerte tal que la inadaptación sería vista como el resultado de ciertas fallas en la economía emocional de la familia. ${ }^{47}$ Para los saberes expertos, la figura de la madre siguió siendo, pese a los cambios, central: durante el "baby boom" de la Segunda Posguerra,

47. ROSE, Nikolas, Governing the soul: the shaping of the private self, Taylor \& Frances/Routledge, London, 1990. las madres mostraron fascinación por los discursos psicológicos -en no pocos casos vehiculizados por médicos- que pretendían explicar los comportamientos del niño y proponían pautas de crianza acordes con la psicología infantil. Pero, al mismo tiempo, se sintieron intimidadas, en la medida en que dichos discursos atribuían un rol central a sus conductas en la conformación de la personalidad de sus hijos. ${ }^{48}$

En el caso que analizamos, la figura de Garrahan representa a una puericultura que daba especial importancia a "lo psicológico", por considerar que numerosos trastornos infantiles se debían a perturbaciones emocionales pasibles de ser explicadas por defectos en el modo como los adultos trataban a los niños. En conexión con esto, el énfasis en la existencia de una individualidad infantil que era necesario respetar venía a cuestionar modelos anteriores basados en el disciplinamiento estricto. Ahora bien, esta forma alternativa de concebir la crianza planteaba mayores exigencias a ambos padres, y sobre todo a la madre. $\mathrm{Al}$ respecto, Garrahan advertía el monto de desconcierto y ansiedad que la "nueva puericultura" podía ocasionar en los padres. En efecto, y a diferencia de la puericultura clásica con sus directivas precisas y consejos categóricos, dejaba un gran margen de elasticidad para comprender y actuar, además de inquietar a los padres con las amenazas de un futuro adverso si el niño no era tratado adecuadamente desde el punto de vista emocional. Frente a esta encrucijada, el autor recomendaba una aplicación flexible y racional de las normas de la "nueva puericultura". Esto suponía ante todo aceptar y disfrutar del hijo tal cual era, teniendo

48 KNIBIEHLER, Yvonne, Historia de las madres..., Op. Cit. 
en cuenta que eventualmente el propio niño podría autorregular su conducta en pos de una mejor adaptación al medio. Sin embargo, esto último no relativizaba la importancia atribuida a la puericultura por su potencial valor para prevenir numerosos trastornos $\mathrm{y}$ promover una mejor salud. Por el contrario, dicha disciplina asumía que si bien el óptimo desarrollo del niño entrañaba el respeto por su individualidad, ello debía darse en un clima de "libertad dentro del orden". ${ }^{49} \mathrm{El}$ horizonte de la "nueva puericultura" propuesta por Garrahan entrañaba, al igual que en la obra de Aráoz Alfaro, el ideal de un sujeto plenamente desarrollado, dueño de atributos tales como la productividad, la autonomía y la capacidad de convivir armónicamente en sociedad. Y en el logro de esta meta la supervisión del médico se volvía imprescindible, no tanto por su valor informativo sino por su capacidad de contener a los padres desde el punto de vista emocional. Hacia mediados del siglo XX, el equilibrio afectivo y la capacidad de comprender al niño parecían ser los mayores desafíos que planteaba a los padres la compleja trama de factores en juego en el desarrollo del niño y su crianza. ${ }^{50}$

\section{Consideraciones finales}

El análisis de las obras de Aráoz Alfaro y Garrahan ha permitido dar cuenta de similitudes y diferencias en los dos momentos analizados, en lo relativo al modo cómo el discurso de la puericultura se dirigió a los padres, conceptualizó el desarrollo infantil y estableció problemas y soluciones en torno a

49. GARRAHAN, Juan Pedro, La Salud del Hijo..., Op. Cit., p. 588.

50. COSSE, Isabella, Pareja, sexualidad y familia en los años sesenta, Siglo Veintiuno, Buenos Aires, 2010. su crianza. En ambos casos, el saber experto interpeló a los padres en tanto responsables del futuro del niño, esperando de su parte una buena predisposición para incorporar las recomendaciones. En este punto, las representaciones de los autores respecto al saber médico hacían hincapié en su rigurosidad científica, hecho que le otorgaba una validez de la que carecían los saberes populares. En el discurso médico, la implementación de estas pautas científicas de crianza contribuiría al pleno desarrollo del niño, que en ambos casos puede reconducirse al ideal de un sujeto autónomo, responsable, productivo y apto para la vida en sociedad. Sin embargo, el tiempo transcurrido entre la publicación de ambas obras introdujo modificaciones importantes que, desde luego, no pueden desligarse de los cambios sociopolíticos que tuvieron lugar durante la primera mitad del siglo XX. En ese sentido, si en Aráoz Alfaro la preocupación central era la supervivencia del niño, su fortaleza física y el porvenir de la especie, es claro que ello se vincula de manera estrecha con el problema acuciante de la mortalidad infantil y con el ideal de progreso de una nación aún en ciernes. De tal modo, la consideración de los aspectos psicológicos del desarrollo desde una matriz naturalista y evolucionista, destacaba ante todo la necesidad de disciplinar la conducta infantil en pos de promover la adaptación al medio. Casi seis décadas después, en un contexto en el cual la tasa de mortalidad infantil había descendido significativamente y las vicisitudes del desarrollo comenzaban a leerse a la luz de la trama relacional que rodeaba al niño, las inquietudes de Garrahan se circunscribían al pleno desenvolvimiento de la personalidad infantil, supeditando el bienestar físico a las variables psicológicas.

Estos cambios a nivel de las formas de 
concebir el desarrollo del niño se plasmaron en el modo como el saber experto incidió en la construcción de representaciones y prácticas en torno a la maternidad y paternidad. Así, hacia los años cincuenta la figura de la madre abnegada en pos de la salud física y moral de su hijo dejaba lugar a aquella otra que veía en el niño la concreción de un deseo compartido con su pareja. Del mismo modo, las motivaciones que regían los deberes maternales habían cambiado en el discurso de la puericultura: el sacrificio personal con el fin de brindar a la patria ciudadanos nobles y trabajadores había dado paso a la compleja tarea de procurar la felicidad del niño y la realización plena de sus potencialidades.

Por último, y en consonancia con estos cambios, la propia puericultura se reconfiguró a lo largo de las décadas. Al igual que en el caso de la pediatría, esta rama de la medicina infantil fue partícipe de un proceso de renovación en virtud del cual se promovió el estudio del niño considerando sus rasgos emocionales y psico-sociales. ${ }^{51}$ Así, la fuerte impronta normativa que caracterizó a la puericultura en sus comienzos fue matizada por un perfil comprensivo, que buscó adaptar las pautas de crianza a los rasgos singulares de cada niño. ${ }^{52}$

Para finalizar podemos decir que el recorrido realizado por dos obras destinadas a la educación de los padres en la Argentina nos permitió explorar la relación que la puericultura estableció con la psicología, en dos sentidos superpuestos: por un lado, la medicina legitimó sus intervenciones echando mano del conocimiento psicológico y oficiando de ese modo como receptora y difusora de teorías psicológicas producidas en otras latitudes. Por otro lado, la forma en que el discurso médico conceptualizó el desarrollo infantil, se plasmó en consejos prácticos por medio de los cuales la puericultura intervino en el proceso de subjetivación de los niños y sus padres, difundiendo un modelo de infancia, de familia y de sociedad "normal". Aun cuando el recorrido realizado en este trabajo dista de ser exhaustivo, creemos haber mostrado de qué modo, en los dos momentos analizados, el saber experto se propuso pautar ciertos modos de vivir, actitudes y valores considerados deseables para el progreso de la nación.

51. ESCARDÓ, Florencio, "La 'Pediatría Psicosomática' como disciplina normativa”, en Archivos Argentinos de Pediatría, Buenos Aires, 1957, V. XLVIII, No 2, pp. 6770.

52. PELUFFO, Euclides y SALDÚN DE

RODRÍGUEZ, María Luisa, "Puericultura coexistencial. Medios docentes y educacionales", en Archivos de Pediatría del Uruguay, Montevideo, 1963, V.XXXIV, No 7, pp. 409-420.

Recibido: 14 - 03 - 2016 Aceptado: 23 - 08 - 2016 Publicado: 30 - 12 - 2016 


\section{Bibliografía}

ARÁOZ ALFARO, Gregorio, El libro de las madres. Pequeño tratado práctico de bigiene del niño con indicaciones sobre el embarazo, parto y tratamiento de los accidentes, Librería científica de Agustín Etchepareborda, Buenos Aires, 1899.

ARÁOZ ALFARO, Gregorio, El Libro de las Madres. Manual práctico de higiene del niño, con indicaciones sobre el embarazo, parto, y tratamiento de los accidentes. Nueva edición reformada, Cabaut y Cía. Editores, Buenos Aires, 1922.

ARMUS, Diego y BELMARTINO, Susana, "Enfermedades, médicos y cultura higiénica", en CATTARUZZA, Alejandro (director) Crisis económica, avance del Estado e incertidumbre politica (19301943), Colección Nueva Historia Argentina, T. VII, Sudamericana, Buenos Aires, 2001.

BIERNAT, Carolina y RAMACCIOTTI, Karina, Crecer y multiplicarse: la politica sanitaria maternoinfantil. Argentina 1900-1960, Biblos, Buenos Aires, 2013.

BILLOROU, María José, "Madres y médicos en torno a la cuna. Ideas y prácticas sobre el cuidado infantil (Buenos Aires, 1930-1945)”, en La Aljaba, Santa Rosa,2007, V.XI, pp. 167-192. Disponible en: <http:// www.scielo.org.ar/pdf/aljaba/v11/v11a08.pdf>

BOLTANSKI, Luc, Puericultura y moral de clase, Editorial Laia, Barcelona, 1974.

BORINSKY, Marcela, “Todo reside en saber qué es un niño. Aportes para una historia de la divulgación de las prácticas de crianza en Argentina”, en Anuario de investigaciones-Psicología UBA, Buenos Aires, 2005, V.XIII, pp. 117-126. Disponible en: <http://www.scielo.org.ar/pdf/anuinv/v13/v13a43.pdf>

BRIOLOTTI, Ana, "Del 'pequeño salvaje' al individuo social: saber médico y desarrollo psicológico en la revista Hijo Mío..!", ponencia presentada en $V$ Congreso Internacional de Investigación y Práctica Profesional en Psicología, Universidad de Buenos Aires, Buenos Aires, 2013. Disponible en: <http:// www.aacademica.org/000-054/117.pdf> [Consulta: 27 octubre 2014].

BRIOLOTTI, Ana y BENÍTEZ, Sebastián, “Medicina, higiene mental y saber psi en la construcción de la maternidad y la infancia en la Argentina: un análisis a través de la revista MADRE Y NIÑO (19341935)", en Universitas Psychologica: Panamerican Journal of Psychology, Bogotá, 2014, V. XIII, n 5, pp. 1709-1719.

BRIOLOTTI, Ana, "La voz de la ciencia educando a los padres: el desarrollo psicológico del niño en las conferencias radiales dirigidas a la comunidad platense (1942-1947)", en VIGUERA, Ariel (coordinador) Historias de la psicología y el psicoanálisis en La Plata (1946-1990), EDULP, La Plata, 2015.

BRIOLOTTI, Ana, "El problema de la inapetencia de origen psíquico en la primera infancia. Un aporte para el análisis de las relaciones entre pediatría, psicología y psicoanálisis en la Argentina (1936-1956)”, ponencia presentada en 4tas Jornadas de Estudios sobre la Infancia: Lo público en lo privado y lo privado en lo público, Universidad Nacional de General Sarmiento, Buenos Aires, 2015, pp. 52-72. Disponible en: $<$ https://www.aacademica.org/4jornadasinfancia/16.pdf> [Consulta: 12 junio 2015]. 
COLÁNGELO, María Adelaida, "El saber médico y la definición de una 'naturaleza infantil' entre fines del siglo XIX y comienzos del siglo XX en la Argentina”, en COSSE, Isabella, LLOBET, Valeria, VILLALTA, Carla y ZAPIOLA, María Carolina (editoras) Infancias: politicas y saberes en Argentina $y$ Brasil: siglos XIX y XX, Teseo, Buenos Aires, 2011.

COSSE, Isabella, Pareja, sexualidad y familia en los años sesenta, Siglo Veintiuno, Buenos Aires, 2010.

COSSE, Isabella, LLOBET, Valeria, VILLALTA, Carla y ZAPIOLA, María Carolina, "Introducción”, en COSSE, Isabella, LLOBET, Valeria, VILLALTA, Carla y ZAPIOLA, María Carolina (editoras) Infancias: politicas y saberes en Argentina y Brasil: siglos XIX y XX, Teseo, Buenos Aires, 2011.

COWEN, Miguel Pablo, "Infancia, abandono y padres en el siglo XIX porteño", en Anuario del Instituto de Historia Argentina, La Plata, 2004, No 4, pp. 75-99. Disponible en: < http://www.fuentesmemoria.fahce. unlp.edu.ar/art_revistas/pr.3249/pr.3249.pdf> [Consulta: 9 de marzo 2014]

DARRÉ, Silvina, Maternidad y tecnologías de género, Katz Editores, Buenos Aires, 2013.

DE LUNA FREIRE, Maria Martha, “Ser mãe é uma ciência': mulheres, médicos e a construção da maternidade científica na década de 1920”, História, Ciências, Saúde-Manguinhos, Rio de Janeiro, 2008, V.XV, suplemento, pp. 153-171.

DE MOURA, Marina, "Saúde e educação na revista Infância”, en Cadernos de História da Ciência. Instituto Butantan, San Pablo, 2010, V. VI, No 2, pp. 27-43. Disponible en: <http://teste.pc2.com.br/butantan/ arquivos/31/PDF/v6n2a02.pdf>

DONZELOT, Jacques, La policía de las familias. Familia, sociedad y poder, Ediciones Nueva Visión, Buenos Aires, 2008.

DOS SANTOS LAMPRECHT, Claudia, “Conselhos às mães: manuais de puericultura como estratégia biopolítica na constituição de infâncias saudáveis e normais”, en TEXTURA-ULBRA, Canoas, 2014, V. XVI, No 32, pp. 210-225. Disponible en: <http://www.periodicos.ulbra.br/index.php/txra/article/ view/1252/942>

ESCARDÓ, Florencio, "Las tesis sobre lactancia”, en Archivos Argentinos de Pediatría, Buenos Aires, 1943, V.XIV, No 6, pp. 448-455.

ESCARDÓ, Florencio, "La 'Pediatría Psicosomática' como disciplina normativa”, en Archivos Argentinos de Pediatría, Buenos Aires, 1957, V. XLVIII, No 2, pp. 67-70.

FOUCAULT, Michel, Los Anormales, Fondo de Cultura Económica, Buenos Aires, 2001.

FOUCAULT, Michel, El Poder Psiquiátrico, Fondo de Cultura Económica, Buenos Aires, 2005.

FOUCAULT, Michel, Seguridad, territorio, población, Fondo de Cultura Económica, Buenos Aires, 2006.

GARRAHAN, Juan Pedro, "Facultad de Ciencias Médicas de Buenos Aires. Cátedra de Clínica Pediátrica y Puericultura. Conferencia inaugural ( $1^{\circ}$ de Octubre de 1942)", en Anales de la Sociedad de Puericultura 
de Buenos Aires, Buenos Aires, 1942, V. VIII, No 4, pp. 368-84.

GARRAHAN, Juan Pedro, "La Universidad argentina y el momento actual", en Archivos de Pediatría del Uruguay, Montevideo, 1945, V.XVI, No 10, pp. 653-54.

GARRAHAN, Juan Pedro, “La nueva Puericultura”, Archivos de Pediatría del Uruguay, Montevideo, 1955, V.XXVI, No 1, pp. 128-134.

GARRAHAN, Juan Pedro, La Salud del Hijo. Puericultura, El Ateneo, Buenos Aires, 1955.

GARRAHAN, Juan Pedro, Antecedentes, Titulos y Trabajos de Pediatría y Puericultura, Universidad de Buenos Aires - Facultad de Ciencias Médicas, Buenos Aires, 1956.

GARRAHAN, Juan Pedro. "Tres años de labor pediátrica extrauniversitaria”, en La Semana Médica, Buenos Aires, 1956, V. LXIII, No 3241, pp. 251-54.

GONZÁLEZ LEANDRI, Ricardo, "Notas acerca de la profesionalización médica en Buenos Aires durante la segunda mitad del siglo XIX”, en SURIANO, Juan (compilador) La cuestión social en Argentina, 18701943, La Colmena, Buenos Aires, 2004.

GONZÁLEZ LEANDRI, Ricardo, "Itinerarios de la profesión médica y sus saberes de Estado. Buenos Aires, 1850-1910”, en PLOTKIN, Mariano y ZIMMERMANN, Eduardo (compiladores) Los saberes del Estado, Edhasa, Buenos Aires, 2012.

GUY, Donna, "Niños abandonados en Buenos Aires (1880-1914) y el desarrollo del concepto de la madre”, en FLETCHER, Lea, Mujeres y cultura en la Argentina del siglo XIX, Feminaria Editora, Buenos Aires, 1994.

JIMÉNEZ LUCENA, Isabel, RUIZ SOMAVILLA, María José y CASTELLANOS GUERRERO, Jesús, "Un discurso sanitario para un proyecto político. La educación sanitaria en los medios de comunicación de masas durante el primer franquismo", en Asclepio, Madrid, 2002; V. LIV, No 1, pp.201-218. Disponible en: <http://asclepio.revistas.csic.es/index.php/asclepio/article/view/126/125>

KNIBIEHLER, Yvonne, Historia de las madres y de la maternidad en Occidente, Nueva Visión, Buenos Aires, 2001.

LOREDO NARCIANDI, José Carlos, "La construcción de niños y niñas en España durante el primer tercio del siglo XX: una primera aproximación genealógica”, en CERRI, Chiara \& SÁNCHEZ CRIADO, Tomás (coordinadores) Edades, transiciones e instituciones, Traficantes de sueños, 2014. Disponible en: <http://e-spacio.uned.es/fez/eserv/bibliuned:500383-IIICongresoEtnografia-1010/Documento.pdf>

MAZZEO, Victoria, "La mortalidad de la primera infancia en la Ciudad de Buenos Aires en el periodo 1860-2002”, en Papeles de Población, México, 2007, V. XIII, No 53, pp. 241-273.

MURILLO, Susana, La ciencia aplicada a politicas sanitarias en Argentina y su relación con la escuela de medicina de la Universidad de Buenos Aires (1869-1905), Tesis de Maestría, Universidad de Buenos Aires, 2001. 
NARI, Marcela, Politicas de maternidad y maternalismo politico, Biblos, Buenos Aires, 2004.

PELUFFO, Euclides y SALDÚN DE RODRÍGUEZ, María Luisa, "Puericultura coexistencial. Medios docentes y educacionales", en Archivos de Pediatría del Uruguay, Montevideo, 1963, V. XXXIV, No 7, pp. 409-420.

RÍOS, Julio César y TALAK, Ana María, "La niñez en los espacios urbanos", en DEVOTO Fernando y MADERO, Marta (editores) Historia de la vida privada en la Argentina, Tomo II: La Argentina plural (1870-1930), Taurus, Buenos Aires, 1999.

ROSE, Nikolas, Governing the soul: the shaping of the private self, Taylor \& Frances/Routledge, London, 1990.

RUSTOYBURU, Cecilia, "Los consejos sobre crianza del Dr. Bonanfant: pediatría, psicoanálisis y escuela nueva (Buenos Aires, fines de la década de 1930)”, en Temas y debates, Rosario, 2012, № 23, pp.103-124. Disponible en: <http://www.temasydebates.unr.edu.ar/index.php/tyd/article/view/31/31>

SULMAN, Michael, "The humanization of the American child: Benjamin Spock as a popularizer of psychoanalytic thought", en Journal of the History of the Behavioral Sciences, New Hampshire, 1973, V. IX No 3, pp. 258-265.

SURIANO, Juan, "Introducción: una aproximación a la definición de la cuestión social en Argentina", en SURIANO, Juan (compilador) La cuestión social en Argentina, 1870-1943, La Colmena, Buenos Aires, 2004.

TALAK, Ana María, "El desarrollo psicológico entre la naturaleza, la cultura y la política (1900-1920)", en GARCÍA, Luciano, MACCHIOLI, Florencia y TALAK, Ana María Psicología, niño y familia en la Argentina 1900-1970: perspectivas históricas y cruces disciplinares, Biblos, Buenos Aires, 2014. 